\title{
New Distortion Measures for Data Compression for Emitter Location
}

\author{
Mark L. Fowler \\ Department of Electrical Engineering \\ State University of New York at Binghamton \\ Binghamton, NY 13902 \\ mfowler@binghamton.edu
}

\begin{abstract}
Data compression methods commonly focus on mean-square error (MSE) distortion. However, in TDOA/FDOA emitter location the distortion measure should focus on parameter accuracy. This paper discusses the proper choice of a distortion measure for this problem. We show that MSE is a poor choice for two reasons: (i) increased MSE may not even effect the parameter accuracy, and (ii) there are other factors that must be considered (e.g., the signal's RMS bandwidth/duration and the relative usefulness of TDOA vs. FDOA). We propose a new measure that captures the true impact of compression and illustrate its importance through a few simple examples.
\end{abstract}

\section{1: Introduction}

An effective way to locate electromagnetic emitters is to measure the time-difference-of-arrival (TDOA) and the frequency-difference-of-arrival (FDOA) between pairs of signals received at geographically separated sites [1]-[3]. The measurement of TDOA/FDOA between these signals is done by coherently cross-correlating the signal pairs [2], [3], and requires that the signal samples of the two signals are available at a common site, which is generally accomplished by transferring the signal samples over a data link from one site to the other site. An important aspect of this that is not widely addressed in the literature is that often the available data link rate is insufficient to accomplish the transfer within the time requirement unless some form of lossy data compression is employed. Furthermore, most of the existing methods for compression for emitter location have focused on using meansquare error (MSE) between the original signal and the decompressed signal as the measure of the distortion introduced by the compression method. This paper shows that MSE is a poor choice for two reasons: (i) increased MSE may not even effect the parameter accuracy, and (ii) there are other factors that must be considered (e.g., the signal's RMS bandwidth/duration and the relative usefulness of TDOA vs. FDOA). A new measure is proposed that captures the true impact of compression and its importance is illustrated through a few simple examples.

The two noisy signals received are complex-valued baseband signals given by

$$
\begin{aligned}
& \hat{s}(k)=s(k)+n(k) \\
& \hat{d}(k)=d(k)+v(k)
\end{aligned}
$$

where $s(k)$ and $d(k)$ are the complex baseband signals of interest and $n(k)$ and $v(k)$ are complex white Gaussian noises, each with real and imaginary parts notated as indicated. The signal $d(k)$ is a delayed and doppler shifted version of $s(k)$. The signal-to-noise ratios (SNR) for these two signals are denoted $S N R$ and $D N R$, respectively (where SNR (non-italic) represents an acronym for signal-to-noise ratio; $S N R$ (italic) represents the SNR for $\hat{s}(k)$, etc.). To cross correlate these two signals one of them (assumed to be $\hat{s}(k)$ here) is compressed, transferred to the other site, and then decompressed before cross-correlation as shown in Figure 1. After lossy compression and decompression signal $\hat{s}_{c}(k)$ has SNR of $S N R_{c}$, and the output SNR after cross-correlation is given by

$$
\begin{aligned}
S N R_{o} & =\frac{W T}{\frac{1}{S N R_{c}}+\frac{1}{D N R}+\frac{1}{S N R_{c} D N R}} \\
& \triangleq W T \times S N R_{e f f}
\end{aligned}
$$

where $W T$ is the time-bandwidth product (or coherent processing gain), with $W$ being the noise bandwidth of the receiver and $T$ being the duration of the received signal [3]; here $S N R_{\text {eff }}$ is defined to be the so-called effective SNR that gets boosted by the coherent processing gain. 
The accuracies of the TDOA/FDOA estimates are bounded by the Cramer-Rao bounds according to

$$
\begin{gathered}
\sigma_{T D O A} \geq \frac{1}{2 \pi B_{r m s} \sqrt{2 S N R_{O}}} \\
\sigma_{F D O A} \geq \frac{1}{2 \pi D_{r m s} \sqrt{2 S N R_{O}}}
\end{gathered}
$$

where $B_{r m s}$ is the signal's RMS (or Gabor) bandwidth in $\mathrm{Hz}, \quad D_{r m s}$ is the signal's RMS (or Gabor) duration in seconds [3]; we will refer collectively to these two signal parameters as the signal's "RMS widths". The RMS bandwidth is given by

$$
B_{r m s}^{2}=\frac{\int f^{2}|S(f)|^{2} d f}{\int|S(f)|^{2} d f}
$$

with $S(f)$ being the Fourier transform of the continuoustime signal $s(t)$ and the RMS duration in seconds is given by

$$
D_{r m s}^{2}=\frac{\int t^{2}|s(t)|^{2} d t}{\int|s(t)|^{2} d t} .
$$

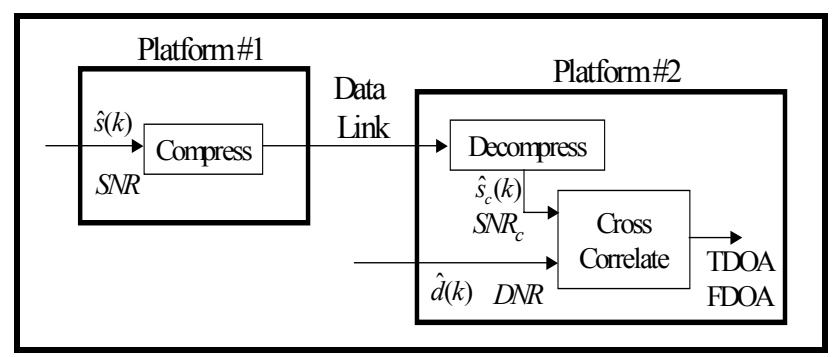

Figure 1: Configuration for processing.

Several results focusing on use of MSE distortion have been published [4]-[7]. For the case of white Gaussian signals and noises, Matthiesen and Miller [4] established bounds on the rate-distortion performance for the TDOA problem and compared them to the performance achievable using scalar quantizers, where distortion is measured in terms of lost SNR due to the mean square error (MSE) of lossy compression. Note that these results are not applicable when locating radar and communication emitters because the signals encountered are not Gaussian. A MSE-based method using block adaptive scalar quantization was proposed by Desjardins [5] and analyzed by Fowler [6] to determine its MSE for various signal types. Wavelet-based methods designed to minimize MSE for a given rate have been proposed [7] and demonstrated [8] to outperform the MSE-based quantization approaches given in [4]-[6].
More recently, though, a new viewpoint has been explored by Fowler [9]-[11] that abandons the sole use of MSE as a distortion measure and strives to exploit the fact that the location accuracy depends on other signal factors that can be exploited during compression. In particular, it is recognized that the location accuracy is impacted by the signal's RMS widths in such a way that a compression method may be able to throw away time-frequency components that have minimal impact on the RMS widths and in so doing realize a significant improvement in compression ratio without significantly reducing the location accuracy. In [9] the idea of using a combination of decimation and quantization was explored: a lowpass filter reduces the signal's bandwidth, enabling data compression through decimation, and then the signal is quantized. The results in [9] determine the optimal balance between quantization and decimation to achieve the best location accuracy - the distortion measure used is the TDOA accuracy, which is impacted by the RMS bandwidth after filtering and the MSE after quantization. It was shown that this approach outperforms the quantization-only and the decimation-only approaches. In [10] and [11], the wavelet transform was used to identify components that contribute insignificantly to the RMS bandwidth and therefore can be discarded by a compression algorithm to gain a significant improvement in compression ratio compared to MSE-based wavelet methods. A particularly striking insight from these papers is that although the decompressed signal obtained from these RMS-based wavelet methods bears very little resemblance to the original signal (i.e., has large MSE), the resulting TDOA accuracy remains remarkably good for such a large MSE.

The results in [9]-[11] all focused on developing algorithms to exploit the non-MSE distortions that arise from looking at the impact of compression on the TDOA accuracy through reduction of its RMS bandwidth and its SNR; the impact of RMS duration on FDOA was not considered in detail. The focus of this paper is not to develop an algorithm but to further explore the characteristics and ramifications of using a non-MSE distortion measure: to demonstrate why the MSE-only distortion measure is poorly suited to emitter location, to consider how to weight the considerations of RMS duration and RMS bandwidth and their impacts on TDOA/FDOA accuracy, and to demonstrate the importance of these considerations via some simple examples. Section II shows why the MSE distortion measure is ineffective and then develops a suitable non-MSE distortion measure, Section III discusses some ramifications nd issues surrounding the use of these new distortion measures, and Section IV discusses the issue of weighting the effects on TDOA and FDOA accuracies using a simple example. 


\section{2: Non-MSE Distortion Measure}

The goodness of a compression scheme is assessed via a rate-distortion analysis - that is, establish a curve that defines the level of distortion as a function of rate. The most common definition of distortion is the mean-square error (MSE) between the signal $\hat{s}(k)$ (see Figure 1) and the de-compressed version of it, $\hat{s}_{c}(k)$. The goal then is to minimize the MSE (or equivalently maximize the $S N R_{\mathrm{c}}$ (see Figure 1)) for a given desired rate. This is also equivalent to maximizing the signal-to-quantization ratio (SQR) of the compression scheme, where $S Q R=P_{s} / P_{n c}$ where $P_{n c}$ is the power of the noise added due to lossy compression. From this we can write

$$
\frac{1}{S N R_{c}}=\frac{1}{S N R}+\frac{1}{S Q R}
$$

and then the output SNR becomes

$$
S N R_{O}=\frac{W T}{\frac{1}{S N R}+\frac{1}{S Q R}+\frac{1}{D N R}+\frac{1}{S N R D N R}+\frac{1}{S Q R D N R}}
$$

from which we can see that as long as $S Q R>$ $\min \{S N R, D N R\}$, the value of $S N R_{o}$ is essentially unaffected by SQR.

Thus, for a compression scheme that relies solely on direct quantization of signal samples (e.g., [5], [6]) the impact of quantization on the TDOA/FDOA accuracy will be invisible until the quantizer is made so coarse that it's SQR drops below a threshold set by $S N R$ and $D N R$. Thus, even in this simple case MSE is not necessarily the best distortion measure to use.

The effect of direct quantization on the TDOA/FDOA accuracy in (3) is solely through its impact on the SNR because quantization cannot effect the signal's RMS widths. However, when the quantization is used as part of some form of transform coding then the quantization could impact the RMS widths. For example, if the transform used is a frequency-domain transform (like the DFT) and certain transform coefficients are set to zero as part of the compression scheme, then this could result in the decompressed signal having a different RMS bandwidth. Similarly, if the transform used is a joint timefrequency transform (like the wavelet transform) and certain transform coefficients are set to zero as part of the compression scheme, then this could result in the decompressed signal having a different RMS duration and RMS bandwidth. Thus, using a pure MSE distortion measure fails to capture the impact of the compression scheme on the TDOA/FDOA accuracy. Thus, a scheme like the wavelet-based method in [7] and [8] that quantizes the wavelet coefficients to achieve a minimum MSE for a given rate fails to fully exploit the signal structure to gain optimal compression performance. Namely, it ignores the fact that some of its coefficients may have little usefulness toward estimating TDOA/FDOA parameters.

Thus, what is needed is a new distortion measure that captures the total effect of a compression scheme on TDOA/FDOA accuracy. The above considerations imply that we should use the TDOA/FDOA accuracies themselves as our distortion measure. We ignore for now the fact that this gives two distortion measures rather than the required single measure. Because cross-correlation methods can achieve the Cramer-Rao bounds [2],[3] we use the right-hand sides of (3) as our distortion measures. Looking at the expressions for TDOA/FDOA accuracies in (3) it is clear that we should take as measures to maximize for a given desired rate the following weighted "RMS-distortion" SNRs:

$$
\begin{aligned}
S N R_{r m s, T D O A} & =B_{r m s}^{2} S N R_{o} \\
& =B_{r m s}^{2}\left(W T \times S N R_{e f f}\right)
\end{aligned}
$$

and

$$
\begin{aligned}
S N R_{r m s, F D O A} & =D_{r m s}^{2} S N R_{o} \\
& =D_{r m s}^{2}\left(W T \times S N R_{e f f}\right)
\end{aligned} .
$$

So the general goal is the following, expressed as transform coding with a non-MSE distortion. Given some signal decomposition

$$
\hat{s}(k)=\sum_{n=1}^{N} c_{n} \psi_{n}(k)
$$

of the signal to be compressed, we wish to select which coefficients should be coded and transmitted to achieve a desired rate-distortion goal where distortion is measured using (8) and (9). For example, we may wish to find a subset $\Omega$ of indices such that the signal given by

$$
\widetilde{s}(k)=\sum_{n \in \Omega} c_{n} \psi_{n}(k)
$$

maximizes (8) and (9) while the set $\left\{c_{n} \mid n \in \Omega\right\}$ can be coded using rate $R$. In general this selection process is quite difficult because of (i) the nonlinear, nonmonotonic relationship between the coefficients and the RMS widths, and (ii) the fact that removing a coefficient from $\Omega$ effects both the RMS widths and $S N R_{o}$. Furthermore, the simultaneous maximization of (8) and (9) can be difficult, especially given that there may be a different ac- 
ceptable level of degradation on TDOA than there is on FDOA; this issue is considered later. A suboptimal approach based on these ideas and using the wavelet transform was presented in [11] where it was shown that the above non-MSE TDOA distortion measure motivates the use of a quadratic weighting along the frequency axis of the wavelet coefficients to emphasize the importance of high frequencies to estimating TDOA.

\section{3: Ramifications of Non-MSE Distortion}

Simpler versions of these non-MSE distortion measures were first used in [9] to find the optimal balance between quantization and decimation for the simple case of a rectangular spectrum - the signal was first lowpass filtered and decimated to reduce the rate and then quantized to reduce the rate further. The rectangular spectrum simplified the scenario so that filtering had no effect on SNR. Here we consider arbitrary spectra and include the effect on SNR. Figure 2a shows that for non-rectangular spectra (as is expected in practice) the drop off in effective SNR $\left(S N R_{r m s, T D O A}\right)$ due to only filtering/decimation is not as damaging as it is for the rectangular case- in fact, for the Gaussian-shaped spectrum the lowpass filtering actually improves the effective SNR because it improves the SNR faster than it degrades the rms bandwidth (up to a point). This result was computed using (8) for the case of an ideal lowpass filter and assuming that any rational decimation rate is achievable. If the signal's highest frequency is $B \mathrm{~Hz}$, it is filtered to $\alpha B$ so the rate reduction factor is $\alpha$.

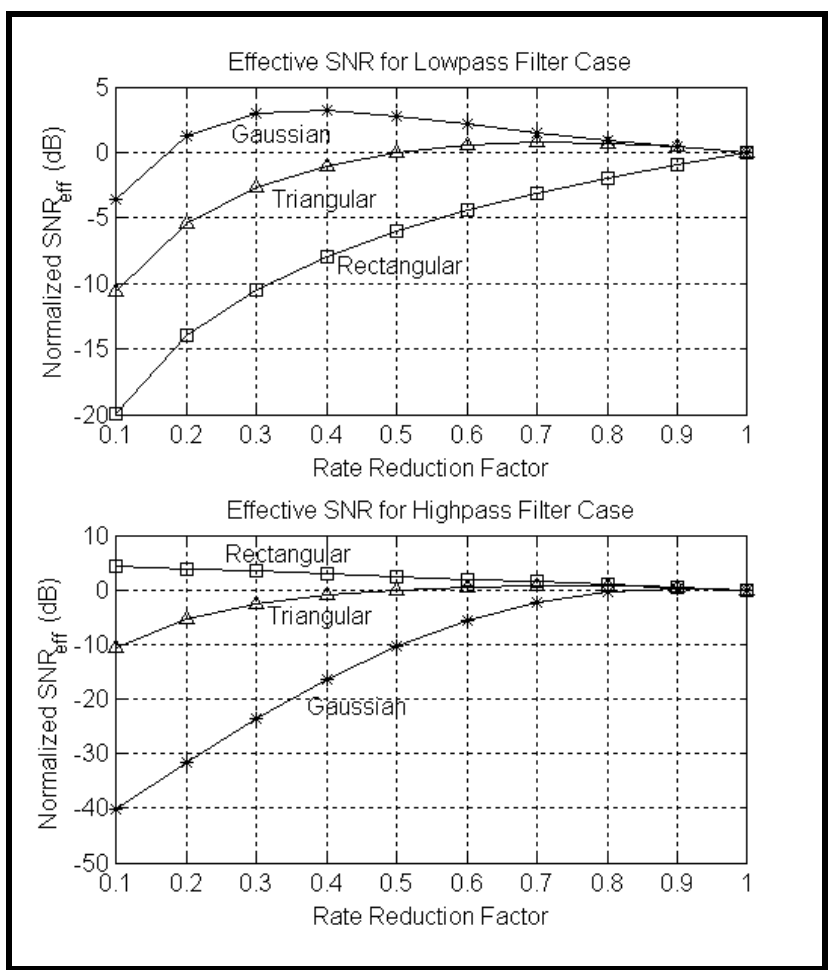

Figure 2: Effective SNR vs. Rate Reduction Factor for SNR $=20 \mathrm{~dB}$.

Of course, it is possible to use filter types other than lowpass in this application. If we apply a highpass filter having cutoff of $(1-\alpha) B \mathrm{~Hz}$, then the bandwidth of the resulting bandpass signal is $\alpha B \mathrm{~Hz}$ and the rate can be reduced by the rate reduction factor is then $\alpha$ (assuming that the signal can be properly bandpass sampled at any value $\alpha$, which won't always be exactly true but will give an insightful result nonetheless). Figure $2 b$ shows the results for this case for the three spectra considered above; note that here it is the rectangular spectrum that yields the improvement as the rate is reduced.

The importance of these results is that they point the way to develop algorithms that exploit the RMS width idea to create very effective compression schemes; in particular, that the impact of compression can in some cases actually improve the accuracy by carefully balancing the RMS bandwidth and SNR considerations. To ensure effective compression the scheme must be tailored to the spectral shape of the signal in a way to balance the effects of noise and RMS widths.

\section{4: TDOA Accuracy vs. FDOA Accuracy}

In Section III we have explicitly considered only TDOA accuracy, or equivalently $S N R_{r m s, T D O A}$. In a general approach we wish to select time-frequency cells in a way that would give high compression but would have minimal joint impact on $S N R_{r m s, T D O A}$ and $S N R_{r m s, F D O A}$. But the 
question then is: what is the right way to combine these two distortion measures into an appropriate single distortion measure? The answer to this question is not at all straight-forward, but has the potential for significant improvements in compression ratios. The answer depends highly on the geometry of the location scenario - thus, if there is some a priori information available about a rough location for the emitter then it can be used to decide how to allocate bits to accordingly. A simple example of this is given. The scenario is shown in Figure 3 here there are four platforms (two pairs) participating to locate the emitter - each pair generates a TDOA/FDOA estimate, which are then fused to find the emitter's location. In this simple 2-D scenario, of course, it is possible to locate the emitter with the TDOA/FDOA estimates from either pair and the location standard deviations can be shown to be

$$
\begin{gathered}
\sigma_{x ; 1,2}=c \sigma_{T D O A ; 1,2} \frac{\sqrt{(B / 2)^{2}+R^{2}}}{B} \\
\sigma_{y ; 1,2}=\lambda \sigma_{F D O A ; 1,2} \frac{\left[(B / 2)^{2}+R^{2}\right]^{3 / 2}}{V B R}
\end{gathered}
$$

for the first pair of platforms and

$$
\begin{gathered}
\sigma_{x ; 3,4}=\lambda \sigma_{F D O A ; 3,4} \frac{\left[(B / 2)^{2}+R^{2}\right]^{3 / 2}}{V B R} \\
\sigma_{y ; 3,4}=c \sigma_{T D O A ; 3,4} \frac{\sqrt{(B / 2)^{2}+R^{2}}}{B}
\end{gathered}
$$

for the second pair of platforms. However, in more realistic scenarios all the platforms would be used to locate the emitter. Thus, we consider here how these two sets of location accuracies combine to determine the location accuracy when all four platforms are used.

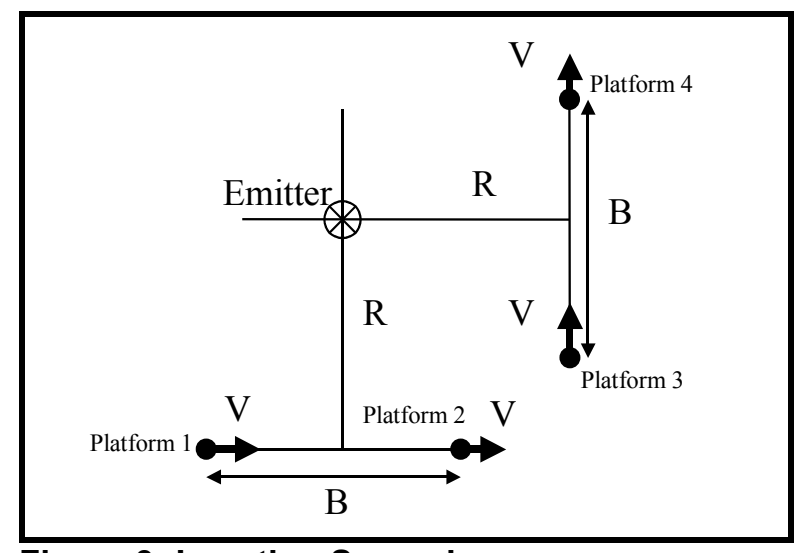

Figure 3: Location Scenario
For the case of $\lambda=0.3 \mathrm{~m}, \mathrm{~B}=10 \mathrm{~km}, \mathrm{R}=70 \mathrm{~km}$, and $\mathrm{V}=200 \mathrm{~m} / \mathrm{s}$ we get $\sigma_{x ; 1,2}=6.3 \mathrm{~m}, \sigma_{y ; 1,2}=740.6 \mathrm{~m}, \sigma_{x ; 3,4}=$ $740.6 \mathrm{~m}$, and $\sigma_{y ; 3,4}=6.3 \mathrm{~m}$. Thus, when using all four platforms together, in this case the FDOA accuracy contributes nothing to the overall location accuracy; therefore, we would be better off compressing to ensure negligible degradation in TDOA accuracy, at the expense of non-negligible degradation in FDOA accuracy. Thus, according to our newly developed distortion measures, we would want to maintain RMS bandwidth but could afford to reduce the RMS duration significantly. This would lead to significantly higher compression ratios than could be accomplished if a pure MSE distortion measure were used.

\section{5: Conclusions}

It is clear that a MSE-only distortion measure is inadequate because it does not properly capture the impact of the compression on the TDOA/FDOA accuracies, especially when the compression is accomplished via timefrequency transform coding. On the other hand, the nonMSE distortion measures introduced here do capture the effect on TDOA/FDOA accuracy because they were derived from considering the relationship between timefrequency RMS widths and the accuracies. As demonstrated here, it is possible to exploit this idea to get improved compression performance. However, as is clear from the considerations in Section IV, it is not straightforward how one should combine the two distortion measures developed, $S N R_{r m s, T D O A}$ and $S N R_{r m s, F D O A}$ into a single measure that would enable the proper balancing of allocation of rate resources between the two measures. This difficulty lies in the fact that to determine the proper allocation depends on knowledge of the geometry between the emitter and the receivers.

\section{6: REFERENCES}

[1] P. C. Chestnut, "Emitter location accuracy using TDOA and differential doppler," IEEE Trans. Aero. and Electronic Systems, vol. AES-18, pp. 214-218, March 1982.

[2] S. Stein, "Differential delay/doppler ML estimation with unknown signals," IEEE Trans. Sig. Proc., vol. 41, pp. 2717 - 2719, August 1993.

[3] S. Stein, "Algorithms for ambiguity function processing," IEEE Trans. Acoust., Speech, and Signal Processing, vol. ASSP-29, pp. 588 - 599, June 1981.

[4] D. J. Matthiesen and G. D. Miller, "Data transfer minimization for coherent passive location systems," Report No. ESD-TR-81-129, Air Force Project No. 4110, June 1981.

[5] G. Desjardins, "TDOA/FDOA technique for locating a transmitter," US Patent \#5,570,099 issued Oct. 29, 1996, Lockheed Martin Federal Systems.

[6] M. L. Fowler, "Coarse quantization for data compression in coherent location systems," IEEE Transactions on Aerospace and Electronic Systems, vol. 36, no. 4, pp. 1269 1278, Oct. 2000. 
[7] M. L. Fowler, "Data compression for TDOA/DD-based location system," US Patent \#5,991,454 issued Nov. 23, 1999, Lockheed Martin Federal Systems.

[8] M. L. Fowler, "Data compression for emitter location," Conference on Information Sciences and Systems, Princeton University, March 15 - 17, 2000, pp. WA7b-14 WA7b-19.

[9] M. L. Fowler, "Decimation vs. Quantization for Data Compression in TDOA Systems," in Mathematics and Applications of Data/Image Coding, Compression, and Encryption III, Mark S. Schmalz, Editor, Proceedings of SPIE
Vol. 4122, pp. 56 - 67, San Diego, CA, July 30 - August 4, 2000.

[10] M. L. Fowler, "Exploiting RMS time-frequency structure for data compression in emitter location systems," National Aerospace and Electronics Conference, Dayton, OH, October 10-12, 2000, pp. $227-234$.

[11] M. L. Fowler, "Non-MSE wavelet-based data compression for emitter location," in Mathematics and Applications of Data/Image Coding, Compression, and Encryption IV, Mark S. Schmalz, Editor, Proceedings of SPIE, San Diego, CA, July 29 - August 3, 2001. 